\title{
A Novel Architecture Based on Cloud Computing for Wireless Sensor Network
}

\author{
Peng Zhang \\ Research Institute of Mobile Internet \\ Xi'an University of Posts and Telecommunications \\ Xi'an, China \\ pzhang@xupt.edu.cn \\ Hanlin Sun \\ School of Compute Science \\ Xi'an University of Posts and Telecommunications \\ Xi'an, China \\ sunhanlin@xupt.edu.cn
}

\author{
Zheng Yan \\ Department of ComNet, \\ Aalto University \\ Espoo, Finland \\ State Key Laboratory of ISN, \\ Xidian University \\ Xi'an, China \\ zheng.yan@aalto.fi, zyan@xidian.edu.cn
}

\begin{abstract}
Wireless sensor network (WSN) is widely applied in many fields since its emergence. However, the limited resources of a sensor, especially limited battery life, limited bandwidth and limited processing power, are the main challenges for deploying and operating WSNs. This paper proposes a novel architecture based on cloud computing for wireless sensor network, which can improve the performance of WSN. Based on this architecture, a cloud acts as a virtual sink with many sink points that collect sensing data from sensors. Each sink point is responsible for collecting data from the sensors within a zone. Sensing data are stored and processed in distributed manner in cloud. Our simulation results show that the proposed architecture improves the performance of WSN, e.g., reduced packet transmission error rate, decreased number of end-to-end hops, and improved efficiency of energy consumption.
\end{abstract}

Keywords-wireless sensor network; data centric network; cloud computing; Hadoop

\section{INTRODUCTION}

Wireless sensor network (WSN) is a self-organizing network consisting of a lot of sensor devices that connect to others through wireless communication channel in multi-hop manner. Sensors collect environment parameters and transmit sensing data back to a central management node (i.e., a sink node) for further processing. Nowadays, WSN has been applied in many fields, such as environment monitoring, military, surveillance, disaster rescue and healthcare etc., and it is more widely used in the Internet of things (IoT) era.

However, sensors are usually low cost devices equipped with limited resources, e.g., processing power, memory, wireless bandwidth, and battery. The design of WSN must take care of these constrains, especially the battery limitation that determines the lifetime of a sensor and a sensor network. In literature, a number of energy efficient techniques have been proposed. All layer protocols used in WSN are optimized to reduce energy consumption, including MAC layer[1], network layer (routing protocols)[2], transport layer[3], and cross-layer approaches[4]. Since transmitting operation consumes more energy comparing against sensing and processing operations, some other technologies have been proposed to save energy, e.g., in-network data processing[5], mobile sink for data collection [6], and topology reorganization [7-8].

Cloud computing is considered as a cost efficient way of IT resource provision manner [9]. It offers a number of advantages such as scalability, agility and economy efficiency, in comparison of traditional IT infrastructure. The cloud computing technology invented by Google is suitable for big data storage and processing. The Hadoop, an open source implementation of Google's cloud by Apache, is widely adopted by many companies.

In this paper, we propose a novel architecture based on cloud computing for wireless sensor network. In this architecture, a cloud acts as a virtual sink and collects sensing data in multiple points. Therefore, the WSN is naturally divided into units which we refer as "zones”. Each node in the cloud is responsible for data collection of sensors in a zone. The sensors in a zone are organized as a local WSN in flat or hierarchy topology, and these local networks are integrated together by the cloud. As a result, the average end-to-end path length of packet transmission could be shortened, and the energy consumption would be reduced. The required bandwidth for data transmission is reduced as well. Moreover, data in cloud are stored and processed in distributed manner, thus complicated tasks could be completed timely, which would be preferred for large volume data process.

The rest of the paper is organized as follows. In section II, we present related work. In section III, we outline our new architecture based on cloud computing. In section IV, we present simulation results and conclude the paper in final section.

\section{RELATED WORK}

Recently, new types of WSN, such as wireless multimedia sensor network (WMSN) and wireless sensor and actor network (WSAN), are emerging. In WMSN, more powerful sensors, for example, image sensor, audio sensor, 
and video sensor, etc., are employed to capture rich information of monitoring area[10]. In WSAN, the actor, which is usually a robust and can move around, is introduced into WSN. During its movement, it is capable of communicating with scalar sensors and reacts to environment changes[11]. In these new sensor networks, the resource challenges (especially bandwidth and energy) are even severer since more data are captured and needed to be processed and transmitted. Data compression, efficient image data coding, cross-layer communication protocols, smart activity scheduling, and energy-efficient routing techniques are typical ways to alleviate problems faced[12-15].

Cloud Computing enables the possibility of wide deployment of rich services in mobile devices. Mobile Cloud Computing (MCC) refers to an infrastructure and mechanisms for providing computation and data storage capabilities to mobile devices based on cloud computing. MCC is seen as the future of mobile [16].

\section{Cloud COMPUting BASEd ARChitecture}

\section{A. Architecture}

The architecture is shown in Fig 1. A number of special nodes (sink points) distributed across the WSN area constitute a cloud. We refer this type node as "cloud node", which is equipped with more resources. Furthermore, a cloud node acts as a sink for sensors nearby. Therefore, the architecture is naturally cluster based. In order to differentiate from the term "cluster" in traditional WSN, we refer a cluster as a "zone" in this paper. These sensors in a zone are organized as independent local WSNs (LWSN), and all LWSN are integrated together by the cloud. The cloud can be viewed as a "virtual sink" for the whole sensor network.

\section{B. Organizing Sensors in a Zone}

We present a different way of heterogeneous sensors organization, as shown in Fig 2. The homogeneous sensors form a WSN (a different type sensor could be used as a

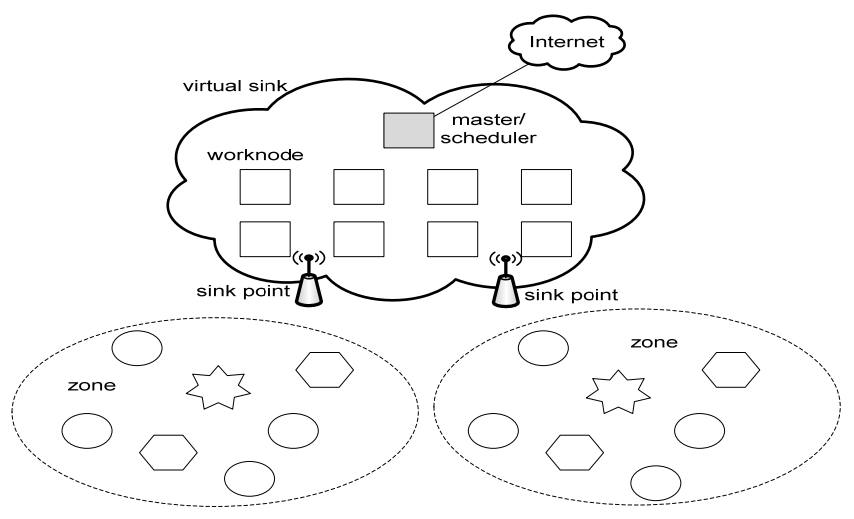

Figure 1. Cloud computing based WSN architecture.

gateway) and send their data to the sink. Each type sensors form a logical independent (and physical overlapped) WSN, and these WSNs access the same sink. Data processing software is deployed in the cloud. After processing data from all sensors, the cloud can designate sensors to perform properly through the accessed sink point. If the size of a zone is reasonable, the scheduling commands from the sink point could reach the destination sensor timely. This way of organization is different from the single-tier clustered architecture of WMSN. First, the independent WSN is built in a zone of which the size is smaller, instead of the whole sensor network. Second, the cloud can control activities of a sensor timely based on data collected from the whole WSN.

\section{Organizing Cloud}

As considering the organization of nodes to a cloud, the bandwidth is still one of the most important factors, especially when they communicate through wireless channel. In [17], Hadoop is adapted to the infrastructure built on smart phones, which has the similar requirements as here. In fact, Hadoop aims for big data storage and process, and data movement reduction is one of its design goals. One of its design criteria is "moving computation is easier than moving data." We suggest the cloud is organized in the Hadoop way. Both of Hadoop's storage system (Hadoop Distributed File System, HDFS) and data processing system (Map-Reduce framework) have a master/slave architecture, in which the master is responsible for storing file meta data or scheduling jobs, and slaves are responsible for storing file content or task execution (task is one piece of split job). The two masters could be deployed either in the same physical node or in different physical nodes. In its storage system, when a client accesses a file, it firstly contacts the master to retrieve the file's meta data (file location, for example), then it directly retrieves the content from the slaves which store a part data of the file. In its data processing system, the code used is submitted to the master, and then the master distributes the code to the slaves which store the processed data, or to those slaves near by the slaves storing the processed data. As a result, the data is processed almost locally and the data movement (thus bandwidth requirement) is reduced. However, data movement cannot be avoided in distributed processing system. Hadoop supports data compression mechanism to reduce bandwidth cost as much as possible. Therefore, a master node should be introduced to

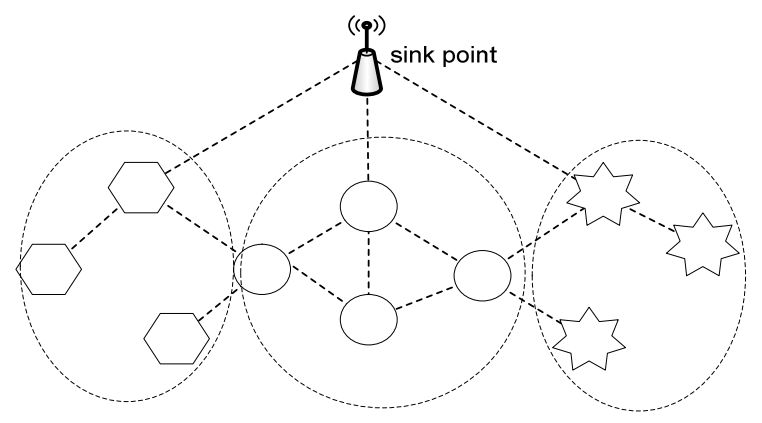

Figure 2. Organizing sensors in a zone.

build the cloud. The master node usually connects to the Internet and is the access point of the system. It should be clear in mind that Hadoop cannot be adopted directly in the 
proposed architecture, just as in Hyrax. The configuration parameters, even the code, should be optimized.

In summary, in the architecture a cloud node is not only a slave of the Hadoop cloud, but also the sink point of a zone. It receives data sent by sensors in the zone, and then acts as a client to store these data into Hadoop. By optimized the Hadoop code, data movement can be avoided, and the client just creates or updates file meta data in master. Data processing requirement and code are sent to the master, and the master schedules the job (the requirement) to run on appropriate slaves. Fig 3 shows an example that cloud nodes communicate with others by Ad-hoc manner to form the virtual sink cloud.

\section{SIMULATIONS}

We perform simulations to verify the performance of WSN in the proposed architecture. Since the performance of the cloud is dependent on the algorithms used in data processing and the communication way hired, we focus only on the performance of packet transmission of sensors. We employ the popular simulator NS2. The scenarios are that sensors are distributed in regular way (grid) in a $200 \mathrm{mX} 200 \mathrm{~m}$ area with each sensor distanced from others in $10 \mathrm{~m}$ in both $\mathrm{X}$ and $\mathrm{Y}$ direction, and there is(are) 1,4 or 8 sink points deployed uniformly in the area. All nodes constitute an Ad-hoc network communicating by IEEE 802.15.4 . The range of radio frequency is $15 \mathrm{~m}$ and the route protocol is AODV. CBR or Poisson traffic generator, which starts randomly in the first 10 seconds of simulation, is deployed in sensors, and each sends packet to the nearest sink point. The packet length is 70 bytes. The CBR generator generates a packet every 5 second, and the average arrival rate of Poisson generator is 5 second as well. All simulations last for 200 seconds.

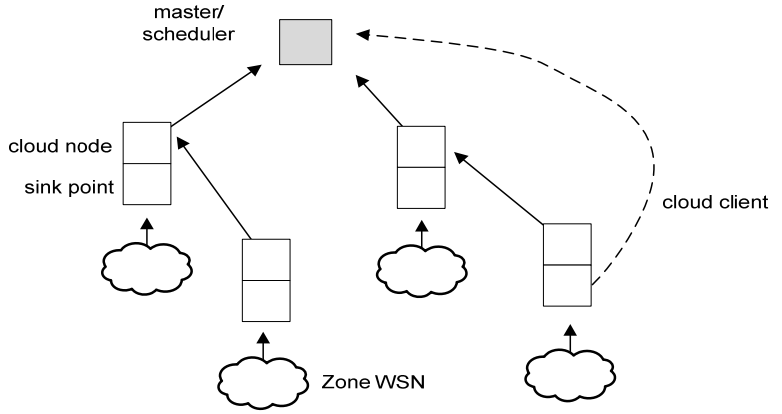

Figure 3. The organization of a cloud, each node in cloud acts as a cloud node and a sink point. Cloud node reaches the master in Ad-hoc manner.

Table 1 and Table 2 show the statistics of simulation results. The last column represents the total number of sending operations of traffic packets divides the number of packets successfully reaches a sink point, and it indicates the efficiency of sending operation thus the energy consumption. Other columns are self explained. It is clear that as the number of sink points increases, the transmission performance of the network is improved greatly. The performance improvement can further be supported by Fig 4 and Fig 5, which are distribution of end-to-end delays and hops, respectively. The smaller delays and the less hops are increased as the number of sink points increases. The delays and hops are of the packets successfully reaching a sink point. In addition, these delays greater than 0.2 second are filtered to make the illustration be clear. The percent of filtered delays is less than $10 \%$.

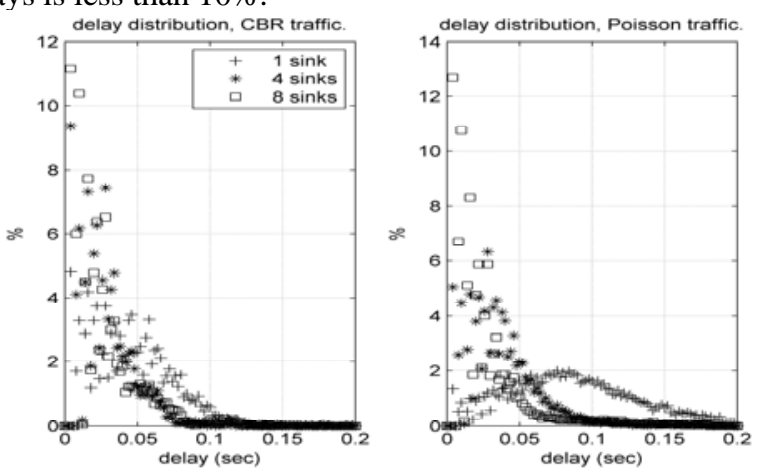

Figure 4. Delay distribution of simulations
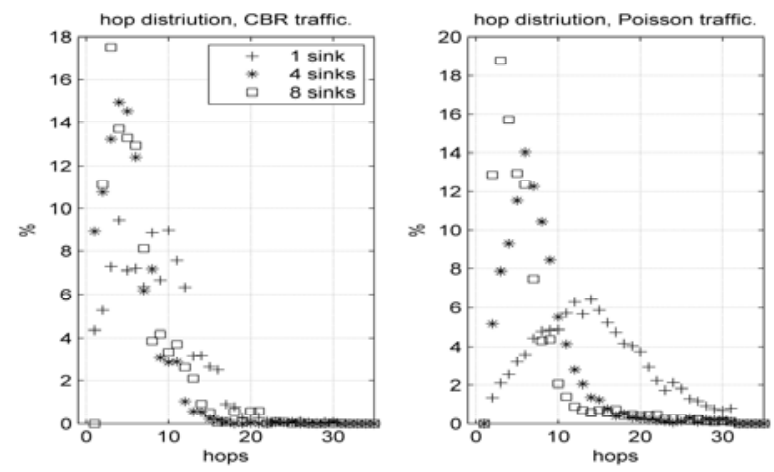

Figure 5. Hop distribution of simulations

\section{CONCLUSIONS}

In this paper, we propose a novel architecture for wireless sensor network based on the cloud computing platform. In the architecture, a number of specific nodes are carefully distributed across the WSN area, and they form a cloud computing platform by Hadoop. The cloud acts as a virtual sink and has multiple sink points which could collect sensing data from WSN. Therefore, the WSN is naturally divided into a number of zones. The sensors in a zone could be organized in flat or hierarchy way as in traditional WSN. We also propose a new way of sensor organization in a zone: the homogeneous sensors form a WSN, while heterogeneous sensors form logical independent but physically overlapped WSNs. Sensors could be managed by the cloud through sink point accessed. All WSNs in zones are integrated together by the cloud. Sensing data in cloud are stored and processed in distributed manner.

Simulations results show that the transmission performance of a WSN built in such architecture is improved greatly. The ratio of packets successfully reaching the sink is increased, the number of end-to-end hops is reduced, and the end-to-end delay is shortened. The average number of sending operations for a packet being sent successfully is 
reduced as well. This indicates that the efficiency of sending operation (thus the energy consumption) is improved. However, the WSN in this architecture is a structured network in which some nodes (at least the cloud nodes) are needed to be carefully managed.

\section{ACKNOWLEDGMENT}

This work is supported by the Fundamental Research Funds for the Central Universities (Grant No. K5051201032), Natural Science Foundation of Education Department of Shaanxi Province, China (Grant No 11JK1018) and Shaanxi International cooperation key project (Grant No. 2012KW03-01).

\section{REFERENCES}

[1] S. H. Gajjar, K. S. Dasgupta, S. N. Pradhan, K. V. Shingala, and K. P. Zinzuwadia, "Comparative analysis of medium access control protocols for wireless wensor networks," in 2011 Annual IEEE India Conference (INDICON), 2011, pp. 1-4.

[2] G. H. Raghunandan and B. N. Lakshmi, "A comparative analysis of routing techniques for wireless sensor networks," in 2011 National Conference on Innovations in Emerging Technology (NCOIET), 2011, pp. 17-22.

[3] L. Su, T. Yan, and L. Yonghua, "A survey of transport protocol for wireless sensor networks," in 2012 2nd International Conference on Consumer Electronics, Communications and Networks (CECNet), 2012, pp. 2338-2341.

[4] S. H. Gajjar, S. N. Pradhan, and K. S. Dasgupta, "Cross layer architectural approaches for wireless sensor networks," in 2011 IEEE Recent Advances in Intelligent Computational Systems (RAICS), 2011, pp. 557-562.

[5] A. Sorniotti, L. Gomez, K. Wrona, and L. Odorico, "Secure and trusted in-network data processing in wireless sensor networks: a survey," Journal of Information Assurance and Security, vol. 2, 2007, pp. 189-199.

[6] W. Bin, X. Dongliang, C. Canfeng, M. Jian, and C. Shiduan, "Employing mobile sink in event-driven wireless sensor networks," in 2008 IEEE Vehicular Technology Conference, 2008, pp. 188-192.
[7] B. Ridong, "Topological optimization based on small world network model in wireless sensor network," in 2011 2nd International Conference on Control, Instrumentation and Automation (ICCIA), 2011, pp. 254-257.

[8] X. Jiu-qiang, W. Hong-chuan, L. Feng-gao, W. Ping, and H. Zhenpeng, "Study on WSN topology division and lifetime," in 2011 IEEE International Conference on Computer Science and Automation Engineering (CSAE), 2011, pp. 380-384.

[9] M. Armbrust. "Above the clouds: a Berkeley view of cloud." University of California, Berkeley. February 2009.

[10] M. AlNuaimi, F. Sallabi, and K. Shuaib, "A survey of wireless multimedia sensor networks challenges and solutions," in 2011 International Conference on Innovations in Information Technology (IIT), 2011, pp. 191-196.

[11] C. Zixing, R. Xiaoping, H. Guodong, C. Baifan, and X. Zhichao, "Survey on wireless sensor and actor network," in 2011 9th World Congress on Intelligent Control and Automation (WCICA), 2011, pp. 788-793.

[12] C. Chen, B. Tian, Y. Li, and Q. Yao, "Data aggregation technologies of wireless multimedia sensor networks: a survey," in 2010 IEEE International Conference on Vehicular Electronics and Safety (ICVES), 2010, pp. 83-88.

[13] X. Zhe-yuan, F. Xiao-ping, L. Shao-qiang, and Z. Zhi, "Distributed image coding in wireless multimedia sensor networks: a survey," in 2010 Third International Workshop on Advanced Computational Intelligence (IWACI), 2010, pp. 618-622.

[14] T. Ma, M. Hempel, D. Peng, and H. Sharif, "A survey of energyefficient compression and communication techniques for multimedia in resource constrained systems," Communications Surveys \& Tutorials, IEEE, vol. PP, 2012, pp. 1-10.

[15] S. Ehsan and B. Hamdaoui, "A survey on energy-efficient routing techniques with QoS assurances for wireless multimedia sensor networks," Communications Surveys \& Tutorials, IEEE, vol. 14, 2012, pp. 265-278.

[16] H. T. Dinh, C. Lee, D. Niyato, P. Wang, "A survey of moible cloud computing -architecture, applications and approaches" in Wireless Communication and Mobile Computing, 2011.

[17] E. E. Marinelli, "Hyrax: cloud computing on mobile devices using mapreduce," Master of Science, School of Computer Science, Carnegie Mellon University, Pittsburgh, 2009.

TABLE I. Statistics OF CBR TRAFFiC SimUlations

\begin{tabular}{|c|c|c|c|c|c|c|c|}
\hline $\begin{array}{c}\text { \# of } \\
\text { sink } \\
\text { points }\end{array}$ & $\begin{array}{c}\text { \# of } \\
\text { generated } \\
\text { packets }\end{array}$ & $\begin{array}{c}\text { \# of } \\
\text { sending } \\
\text { operations }\end{array}$ & $\begin{array}{c}\text { \# of } \\
\text { successful } \\
\text { sending } \\
\text { packets }\end{array}$ & $\begin{array}{c}\text { success } \\
\text { ratio }\end{array}$ & $\begin{array}{c}\text { avg. hops } \\
\text { per } \\
\text { success } \\
\text { packet }\end{array}$ & $\begin{array}{c}\text { avg. delay } \\
\text { per success } \\
\text { packet } \\
\text { (sec) }\end{array}$ & $\begin{array}{c}\text { avg. \# of } \\
\text { sending } \\
\text { operations per } \\
\text { success packet }\end{array}$ \\
\hline 1 & 5336 & 44444 & 3588 & $67.24 \%$ & 8.03 & 0.2861 & 12.39 \\
\hline 4 & 4424 & 24010 & 3558 & $80.43 \%$ & 5.10 & 0.2054 & 6.75 \\
\hline 8 & 10571 & 54552 & 10394 & $97.90 \%$ & 4.95 & 0.1056 & 5.25 \\
\hline
\end{tabular}

TABLE II. Statistics OF POISSON TRACFFIC SimUlations

\begin{tabular}{|c|c|c|c|c|c|c|c|}
\hline $\begin{array}{c}\text { \# of } \\
\text { sink } \\
\text { points }\end{array}$ & $\begin{array}{c}\text { \# of } \\
\text { generated } \\
\text { packets }\end{array}$ & $\begin{array}{c}\text { \# of } \\
\text { sending } \\
\text { operations }\end{array}$ & $\begin{array}{c}\text { \# of } \\
\text { successful } \\
\text { sending } \\
\text { packets }\end{array}$ & $\begin{array}{c}\text { success } \\
\text { ratio }\end{array}$ & $\begin{array}{c}\text { avg. hops } \\
\text { per } \\
\text { success } \\
\text { packet }\end{array}$ & $\begin{array}{c}\text { avg. delay } \\
\text { per success } \\
\text { packet } \\
\text { (sec) }\end{array}$ & $\begin{array}{c}\text { avg. \# of } \\
\text { sending } \\
\text { operations per } \\
\text { success packet }\end{array}$ \\
\hline 1 & 16711 & 247622 & 10873 & $65.06 \%$ & 13.02 & 0.0892 & 14.82 \\
\hline 4 & 13569 & 94566 & 12674 & $93.40 \%$ & 6.40 & 0.0399 & 7.46 \\
\hline 8 & 12168 & 63837 & 11499 & $94.50 \%$ & 4.87 & 0.0297 & 5.55 \\
\hline
\end{tabular}

